\title{
Increased urinary L-histidine in patients with asthma-COPD overlap: a pilot study
}

This article was published in the following Dove Press journal: International Journal of COPD

\author{
Jee Youn Oh' \\ Young Seok Lee' \\ Kyung Hoon Min' \\ Gyu Young Hur' \\ Sung Yong Lee' \\ Kyung Ho Kang' \\ Chin Kook Rhee ${ }^{2}$ \\ Seoung Ju Park ${ }^{3}$ \\ Adnan Khan ${ }^{4}$ \\ Jinhyuk $\mathrm{Na}^{4}$ \\ Youngja H Park ${ }^{4}$ \\ Jae Jeong Shim'
}

'Division of Pulmonary, Allergy and Critical Care Medicine, Department of Internal Medicine, Korea University

Guro Hospital, Seoul, Republic

of Korea; ${ }^{2}$ Division of Pulmonary

Medicine, Department of Internal

Medicine, Catholic University Seoul

Hospital, Seoul, Republic of Korea;

${ }^{3}$ Division of Pulmonary Medicine,

Department of Internal Medicine,

Chonbuk National University

Hospital, Jeonju, Republic of Korea;

${ }^{4}$ Metabolomics Laboratory, College of

Pharmacy, Korea University, Sejong,

Republic of Korea

Correspondence: Jae Jeong Shim

Division of Pulmonary, Allergy and

Critical Care Medicine, Department of

Internal Medicine, Korea University Guro

Hospital, Korea University College of

Medicine, 148 Gurodong-ro, Guro-gu,

Seoul 08308, Republic of Korea

Tel +82 I0 38496639

Fax +8222626 II 66

Email jaejshim@kumc.or.kr

Youngja H Park

Metabolomics Laboratory, College of

Pharmacy, Korea University, Sejong

30019 , Republic of Korea

$\mathrm{Tel}+8244860$ I62।

Fax +82 $44860 \quad$ I607

Email yjhwang@korea.ac.kr
Purpose: Asthma-COPD overlap (ACO) is heterogeneous in nature and requires a unified diagnostic approach. We investigated the urinary levels of L-histidine, a precursor of histamine related to inflammatory responses, as a new candidate biomarker for diagnosing this condition.

Patients and methods: We performed a prospective multicenter cohort study with retrospective analysis of 107 patients, who were divided into three groups: asthma, COPD, and ACO, according to the Spanish guidelines algorithm. Urinary L-histidine levels were measured using liquid chromatography-mass spectrometry. High-resolution metabolomic analysis, coupled with liquid chromatography-mass spectrometry and followed by multivariate statistical analysis, was performed on urine samples to discriminate between the metabolic profiles of the groups.

Results: Urinary L-histidine levels were significantly higher in patients with ACO than in those with asthma or COPD, but the subgroups of ACO, classified according to disease origin, did not differ significantly. High urinary L-histidine level was a significant factor for the diagnosis of ACO even after adjusting for age, sex, and smoking amount. Among patients with airflow obstruction, the urinary L-histidine levels were elevated in patients with a documented history of asthma before the age of 40 years or bronchodilator responsiveness $\geq 400 \mathrm{~mL}$; bronchodilator responsiveness $\geq 200 \mathrm{~mL}$ of forced expiratory volume in 1 second and exceeding baseline values by $12 \%$ on two or more visits; blood eosinophil count $\geq 300$ cells $\cdot \mathrm{mm}^{-3}$; and frequent exacerbations $(P<0.05)$.

Conclusion: Urinary L-histidine could be a potential biomarker for ACO, regardless of the diversity of diagnostic definitions used.

Keywords: asthma, COPD, ACO, urinary L-histidine, metabolomics, inhaled corticosteroid

\section{Introduction}

Asthma-COPD overlap (ACO) was initially described as a condition characterized by an increased variability in airflow associated with an incompletely reversible airflow obstruction. ${ }^{1}$ However, there is no universally accepted definition for this condition, and this leads to diverse epidemiology and outcomes of ACO. ${ }^{2}$ Indeed, ACO is not a single disease, but a combination of syndromes, consisting of several different endotypes and phenotypes with diverse pathogeneses. ${ }^{3}$ Classification based on the inflammatory profiles and treatable traits, irrespective of taxonomy, would help provide more personalized treatment. However, the identification of new biomarkers for treatable symptoms is needed. ${ }^{4}$

The main reason for diagnosing ACO in clinical practice is to identify patients with COPD who are likely to have a better response to inhaled corticosteroids (ICSs). ${ }^{4,5}$ Recently, the use of a Th-2 inflammatory signature (defined by a blood eosinophil count $\geq 300$ cells $\mu \mathrm{L}^{-1}$ and/or a sputum eosinophil count $\geq 3 \%$ ) and genomic signatures 
of Th-2 inflammation, instead of historical classifications of chronic obstructive airway disease (COAD), was suggested to guide ICS therapy. ${ }^{6,7}$ However, the cutoff points of blood eosinophil counts are arbitrary, and other Th-2 signatures are difficult to measure. Thus, more markers that represent Th-2 features are required to distinguish ACO for clinical applications.

Histamine is important in the inflammatory response and airway smooth muscle contraction. ${ }^{8,9}$ Patients with ACO might secrete higher levels of histamine than patients with asthma or COPD, because of airway hyper-responsiveness, ${ }^{10,11}$ repetitive Th-2-related inflammation, and smooth muscle contraction. ${ }^{11,12}$ Moreover, urine is easy to collect and has a high concentration of metabolites, ${ }^{13}$ which are important indicators of pathological states. ${ }^{14,15}$

Thus, we investigated urinary L-histidine, a precursor of histamine, as a new candidate for the diagnosis of ACO by using high-resolution metabolomics (HRM) and liquid chromatography-mass spectrometry (LC-MS), which is an emerging technique to measure small molecules $<1,000 \mathrm{Da}$ in given samples simultaneously and to determine the important features associated with diseases.

\section{Patients and methods Study design}

We performed this prospective cohort study for developing optimal clinical and biological markers for the diagnosis and monitoring of treatment response in patients with chronic airway disease. This multicenter study was performed in Korea University Guro Hospital, Seoul St Mary’s Hospital, and Chonbuk National University Hospital and used the airway disease registry from June 2015 to May 2016. The inclusion criteria for the cohort were as follows: adult patients aged $>19$ years and patients clinically diagnosed with asthma, COPD, or ACO who had been in a stable state without exacerbation and worsening of symptoms for at least 3 months. Patients with all other diseases that could cause airflow obstruction, including bronchiectasis, tuberculosisdestroyed lungs, or interstitial lung disease, were excluded.

The patients were divided into three groups based on age, medical history, smoking history, pulmonary function test (PFT) results, and blood eosinophil count. COPD $(n=38)$ was diagnosed on the basis of the American Thoracic Society and Global Initiative for Chronic Obstructive Lung Disease guidelines, ${ }^{16}$ ie, airflow limitation confirmed by post-bronchodilator spirometry, with forced expiratory volume in 1 second $\left(\mathrm{FEV}_{1}\right) /$ forced vital capacity $(\mathrm{FVC})<70 \%$ and a history of smoking or biomass exposure. Asthma $(n=32)$ was defined according to the Global Initiative for Asthma guidelines. ${ }^{17}$
The diagnosis of ACO $(n=37)$ was based on the GesEPOC (the Spanish COPD guidelines) and GEMA (the Spanish Guidelines for the Management of Asthma) algorithms. ${ }^{18}$ Patients aged $>35$ years with significant exposure to smoking ( $\geq 10$ pack-years), persistent airflow limitation, and with a current diagnosis of asthma or with a significantly positive

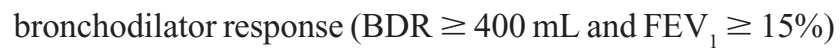
and/or significant blood eosinophilia ( $\geq 300$ cells $\cdot \mathrm{mm}^{-3}$ ) were included in the ACO group.

The following variables were evaluated: smoking history and amount, body mass index (BMI), modified Medical Research Council (mMRC) dyspnea score, St George Respiratory Questionnaire (SGRQ) score, COPD assessment test (CAT) score, asthma control test (ACT) score, PFT (spirometry, lung volume, and diffusing capacity of the lung for carbon monoxide $\left[\mathrm{DL}_{\mathrm{CO}}\right]$ ) results, fractionated exhaled nitric oxide (FeNO; ppb), and exacerbation history for 1 year. Blood tests, including eosinophil counts, were performed routinely on the day of enrollment. Urine samples in the overnight-fasted and medication-free state were collected.

FeNO measurement was performed online by using the NIOX MINO ${ }^{\circledR}$ instrument (Aerocrine AB, Solna, Sweden) at $50 \mathrm{~mL} \mathrm{~min}{ }^{-1}$. An exacerbation was defined as a sustained worsening of the patient's condition, from the stable state and beyond normal day-to-day variations, that is acute in onset and necessitates a change in regular medication with an unscheduled Dr's visit because of the worsened respiratory symptoms. Moderate exacerbation was defined as an increase in symptoms that requires treatment with antibiotics and/or corticosteroids, and severe exacerbation was defined as one that requires hospitalization. Patients with two or more moderate-to-severe exacerbations within 1 year and with one or more hospitalizations for severe exacerbation were defined as frequent exacerbators. A significantly positive BDR was defined as $\geq 400 \mathrm{~mL}$ and high eosinophilia was defined as blood eosinophil count $\geq 300$ cells $\mu \mathrm{L}^{-1}$.

The study protocol was approved by the institutional review boards (Korea University Guro Hospital: KUGH 13246; Seoul St Mary's Hospital: KC15OIMI0553; and Chonbuk National University Hospital: 2015-01-018-005). Written informed consent was provided by the patients, and the study conformed to the tenets of the Declaration of Helsinki.

\section{HRM coupled with LC-MS}

For HRM analyses, $50 \mu \mathrm{L}$ of sample aliquots was treated with acetonitrile (Burdick \& Jackson, Muskegon, MI, USA) $(1: 4, \mathrm{v} / \mathrm{v})$ and centrifuged at $13,000 \times g$ for $10 \mathrm{~min}$ at $4^{\circ} \mathrm{C}$ to separate proteins. ${ }^{19,20}$ Urine samples were analyzed using 
Agilent 1290 Infinity ultra performance liquid chromatography (UPLC) (Agilent Technologies, Santa Clara, CA, USA) connected to a 6550 iFunnel Q-ToF LC-MS with an electrospray ionization (ESI) source (Agilent Technologies). Samples were analyzed in the ESI-positive ion mode. Mobile phases were high-performance liquid chromatography (HPLC)-grade water (Tedia, Fairfield, OH, USA) with $0.1 \%$ formic acid (Fluka, St Louis, MO, USA) (A) and HPLCgrade acetonitrile (Burdick \& Jackson) with $0.1 \%$ formic acid (Fluka) (B). UPLC gradient conditions were as follows: 0.0-1.0 $\min 95 \% \mathrm{~A} ; 1.0-9.0 \mathrm{~min} 55 \% \mathrm{~A} ; 9.0-12.0 \mathrm{~min}$ $10 \% \mathrm{~A} ; 12.0-13.5 \mathrm{~min} 10 \% \mathrm{~A}$; and $13.5-13.6 \mathrm{~min} 95 \% \mathrm{~A}$ at a flow rate of $0.4 \mathrm{~mL} \mathrm{~min}^{-1}$. The injection volume was $3 \mu \mathrm{L}$, and each sample was randomly analyzed in triplicate. The analytical column was a Hypersil GOLD aQ (Thermo Fisher Scientific, Waltham, MA, USA; $1.9 \mu \mathrm{m}, 100 \times 2.1 \mathrm{~mm}^{2}$ ) maintained at $45^{\circ} \mathrm{C}$. The gas temperature was $250^{\circ} \mathrm{C}$, and the nebulizer pressure was 35 psig. The flow rates of the drying gas and sheath gas were $14 \mathrm{~L} \mathrm{~min}^{-1}$ and $11 \mathrm{~L} \mathrm{~min}^{-1}$, respectively. Mass spectra, ranging from a mass-to-charge ratio $(\mathrm{m} / \mathrm{z})$ of 50-1,000, were collected with 20,000 resolutions over 15 min LC runs. ${ }^{21}$

\section{Data preprocessing and extraction of mass spectral data}

Mass spectral data acquired were converted to mzXML by using ProteoWizard (http://proteowizard.sourceforge.net/) followed by adaptive processing of high-resolution LC-MS data (apLCMS) to obtain ion intensities. ${ }^{21}$ The apLCMS provided $5,213 \mathrm{~m} / \mathrm{z}$ within a range of ions set from 50 to 1,000. Data were further processed with normalization, scaling, filtering, and statistical analysis using MetaboAnalyst 3.0 (www.metaboanalyst.ca), which is a web server designed to analyze metabolomic data comprehensively for visualization and interpretation. MetaboAnalyst improves the statistical analysis by filtering the non-informative variables that are unlikely to be of use for further modeling of data, by applying an interquartile range (IQR). In preprocessing mass spectral data, data sets were quantile normalized, log transformed, and pareto-scaled to obtain more comparable individual features before carrying out statistical analyses.

\section{Determination of metabolic profiles and identification of potential metabolites of ACO from asthma and COPD}

Metabolites altered between each pair of groups were considered important toward the identification of potential metabolites related to the metabolic effects of ACO that were different from those of asthma and COPD. Accurate masses of significant metabolites were searched against the online biochemical database service The Human Metabolome Database (http://www.hmdb.ca/). ${ }^{22}$

\section{Statistical analysis}

Clinical data were presented as the median and IQR for continuous variables and as a percentage for categorical variables. Data were compared using the Kruskal-Wallis test for continuous variables, comparing each two groups using the Mann-Whitney $U$-test with Bonferroni corrections ( $\alpha<0.017$; significant). For categorical variables, Pearson's $\chi^{2}$ test or Fisher's exact test was used. Correlations between plasma biomarkers and parameters were analyzed using Spearman's correlation analysis. Significance was defined as $P<0.05$. All analyses were conducted using IBM SPSS Statistics for Windows, Version 20.0 (IBM Corporation, Armonk, NY, USA). Student's $t$-test and orthogonal signal correction partial least squares-discriminant analysis (OPLS-DA) were performed using MetaboAnalyst 3.0, to identify the differentially expressed metabolites among the comparison groups. The intensity of L-histidine in the compared groups was analyzed using GraphPad Prism v 5.03 (GraphPad Software, Inc., La Jolla, CA, USA).

\section{Results}

\section{Patient characteristics}

A total of 107 patients were enrolled. Their baseline characteristics are described in Table 1. Patients with asthma were significantly younger than patients with COPD. Most patients in the COPD and ACO groups were male. All of the patients with COPD and ACO were smokers by definition. Comorbidities other than allergic rhinitis were not significantly different between the groups. The mMRC and SGRQ scores of patients with COPD were higher than those of patients with asthma. In the PFTs, BDR was the highest in patients with ACO. Median serum creatinine levels (asthma, 0.8 [0.6-0.9] mg dL ${ }^{-1}$; COPD, 0.8 [0.6-0.9] $\mathrm{mg} \mathrm{dL}^{-1}$; ACO, 0.7 [0.6-0.8] $\mathrm{mg} \mathrm{dL}^{-1} ; P=0.167$ ) and creatinine clearance rates (asthma, 100.5 [71.5-120.9] $\mathrm{mL} \mathrm{min}^{-1}$; COPD, 95.7 [66.4-123.0] mL min ${ }^{-1}$; ACO, 95.7 [66.4-122.9] $\mathrm{mL} \mathrm{min}^{-1}$; $P=0.606)$ were similar among the groups.

\section{Urine metabolomics}

To observe the urinary metabolic alterations among asthma, COPD, and ACO, we compared the three groups through multiple testing, by inserting the apLCMS feature table containing 5,213 features obtained from 107 urine samples. Student's $t$-test was first utilized to observe the difference in 
Table I Comparison of the baseline characteristics of chronic inflammatory airway diseases

\begin{tabular}{|c|c|c|c|c|}
\hline Parameters & $\begin{array}{l}\text { Asthma } \\
\mathrm{n}=32\end{array}$ & $\begin{array}{l}\text { COPD } \\
n=38\end{array}$ & $\begin{array}{l}\text { ACO } \\
n=37\end{array}$ & $P$-value \\
\hline Age, years & $57.0(47.0-66.8)^{\mathrm{a}}$ & $67.0(61.5-74.5)$ & $64.0(57.0-7 \mid .5)$ & 0.001 \\
\hline Sex, male, n (\%) & $12(37.5)$ & $37(97.4)$ & $33(89.2)$ & $<0.001$ \\
\hline $\mathrm{BMI}\left(\mathrm{kg} \mathrm{m}^{-2}\right)$ & $23.2(21.5-26.9)$ & $22.3(20.6-23.9)$ & $24.6(21.3-27.3)$ & 0.039 \\
\hline Smoker, n (\%) & $3(9.4)^{\mathrm{a}}$ & $38(100.0)$ & $37(100.0)^{c}$ & $<0.001$ \\
\hline Ex-smoker, n (\%) & $\mathrm{I}(3.1)$ & $17(44.7)$ & $23(62.2)$ & \\
\hline Current smoker, n (\%) & $2(6.3)$ & $21(55.3)$ & $14(37.8)$ & \\
\hline Smoking amount (pack-years) & $0.0(0.0-0.0)^{\mathrm{a}}$ & $40.0(33.8-54.8)$ & $35.0(20.0-44.5)^{\mathrm{c}}$ & $<0.001$ \\
\hline \multicolumn{5}{|l|}{ Comorbidities, n (\%) } \\
\hline Allergic rhinitis & $19(59.4)^{\mathrm{a}}$ & $2(5.3)^{b}$ & $17(45.9)$ & $<0.001$ \\
\hline Heart diseases & $12(37.5)$ & $16(42.1)$ & $16(43.2)$ & 0.585 \\
\hline \multicolumn{5}{|l|}{ Symptom scores } \\
\hline $\mathrm{mMRC}$ & $0.0(0.0-1.0)^{\mathrm{a}}$ & $1.0(0.0-2.0)$ & $0.0(0.0-1.0)$ & 0.015 \\
\hline CAT & - & $14.0(10.0-22.0)$ & $12.0(9.5-16.8)$ & 0.050 \\
\hline ACT & $23.0(21.0-24.0)$ & - & $20.0(14.0-22.3)^{\mathrm{c}}$ & 0.001 \\
\hline SGRQ & $10.5(5.1-15.0)^{\mathrm{a}}$ & $16.9(11.0-32.6)$ & $15.3(8 . \mid-23.6)$ & 0.008 \\
\hline \multicolumn{5}{|l|}{ PFTs } \\
\hline $\mathrm{FEV}_{1}, \mathrm{~L}$ & $2.6(2.0-2.8)^{\mathrm{a}}$ & $1.7(1.0-2.5)$ & $1.9(1.6-2.4)^{c}$ & $<0.001$ \\
\hline $\mathrm{FEV}_{1}, \%$ & $91.0(78.3-102.8)^{\mathrm{a}}$ & $66.5(35.8-76.3)$ & $65.0(49.0-71.5)^{\mathrm{c}}$ & $<0.001$ \\
\hline $\mathrm{FEV}_{1} / \mathrm{FVC}, \%$ & $76.0(71.3-80.8)^{\mathrm{a}}$ & $54.0(34.8-58.3)$ & $56.0(44.5-64.5)^{c}$ & $<0.001$ \\
\hline FEF $25 \%-75 \%$ & $64.5(47.0-92.8)^{\mathrm{a}}$ & $21.5(10.8-28.5)$ & $27.0(16.5-37.0)^{c}$ & $<0.001$ \\
\hline $\mathrm{BDR}, \mathrm{mL}$ & $105.0(32.5-150.0)$ & $50.0(|7.5-| 42.5)^{\mathrm{b}}$ & $290.0(200.0-400.0)^{c}$ & $<0.001$ \\
\hline TLC, \% & $10 \mid .5(94.3-108.5)^{\mathrm{a}}$ & $108.5(102.8-117.0)$ & $110.0(103.5-116.0)^{c}$ & 0.013 \\
\hline FRC, \% & $107.0(92.3-123.0)^{\mathrm{a}}$ & $14 \mid .5(|28.8-| 57.0)$ & $130.0(\mid 10.0-149.5)^{c}$ & $<0.001$ \\
\hline $\mathrm{RV}, \%$ & $103.5(86.3-125.8)^{\mathrm{a}}$ & $142.0(\mid 32.8-155.8)$ & $138.0(\mid 20.5-153.0)^{c}$ & $<0.001$ \\
\hline $\mathrm{DL}_{\mathrm{co}}, \%$ & $97.0(90.3-110.8)^{\mathrm{a}}$ & $69.5(47.5-98.8)$ & $90.0(67.5-98.5)^{c}$ & $<0.001$ \\
\hline FeNO, ppb & $22.0(17.0-35.0)$ & I8.0 (II.5-25.5) & $20.0(10.3-35.8)$ & 0.198 \\
\hline Frequent exacerbator, n (\%) & I (3.1\%) & $5(13.2 \%)$ & $6(16.2 \%)$ & 0.204 \\
\hline
\end{tabular}

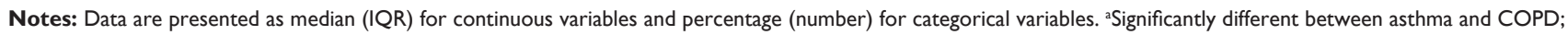
bsignificantly different between COPD and ACO; 'significantly different between asthma and ACO.

Abbreviations: ACO, asthma-COPD overlap; ACT, asthma control test; $B D R$, bronchodilator response; $B M I$, body mass index; $C A T$, COPD assessment test; $D L_{\text {co, }}$ diffusing capacity of the lung for carbon monoxide; FEF, forced expiratory flow; FeNO, fractionated exhaled nitric oxide; FEV , forced expiratory volume in I second; FRC, functional residual capacity; FVC, forced vital capacity; IQR, interquartile range; mMRC, modified Medical Research Council; PFTs, pulmonary function tests; RV, residual volume; SGRQ, St George Respiratory Questionnaire; TLC, total lung capacity.

the urinary metabolic phenotype of ACO in comparison with asthma and COPD. Of the 5,213 features, 223 were found significant in the $t$-test $(P<0.05)$ between the ACO and asthma group urine samples, whereas 215 features were significantly different between the ACO and COPD group urine samples. Furthermore, to better define the metabolic alteration among the three groups and to visualize the variation among groups, we performed OPLS-DA. In OPLS-DA, the entire sample cluster of ACO showed a clear separation from the clusters of asthma (Figure 1A) and COPD (Figure 1B). Such an apparent separation of ACO in comparison with asthma and COPD indicated that the metabolic profile of ACO was highly differentiated from that of asthma and COPD. Moreover, the results of the cross-validation procedure indicated that neither models were overfitting and that both models were reliable, as shown in Figure 1A (intercepts: $R^{2}=0.964$ and $Q^{2}=0.153$ ) and Figure 1B (intercepts: $R^{2}=0.971$ and $Q^{2}=0.046$ ). Asthma and COPD urine samples also showed a similar separation pattern in OPLS-DA, as shown in Figure $1 \mathrm{C}$ (intercepts: $R^{2}=0.963$ and $Q^{2}=0.163$ ).

After observing such variations in the metabolome of ACO, we desired to analyze the urinary level of L-histidine in ACO compared with that in asthma and COPD. Interestingly, as shown in Figure 2A, the urinary level of L-histidine ( $\mathrm{m} / \mathrm{z}$ 178.0571, $[\mathrm{M}+\mathrm{Na}]^{+}$) was significantly higher in patients with ACO than in those with asthma and COPD. Furthermore, to confirm that L-histidine was specifically elevated among patients with ACO, we analyzed the urine samples of patients with asthma and compared them with those of patients with COPD. Although the OPLS-DA showed a clear separation between the two groups (Figure 1C), the urinary level of L-histidine was not significantly different among patients 

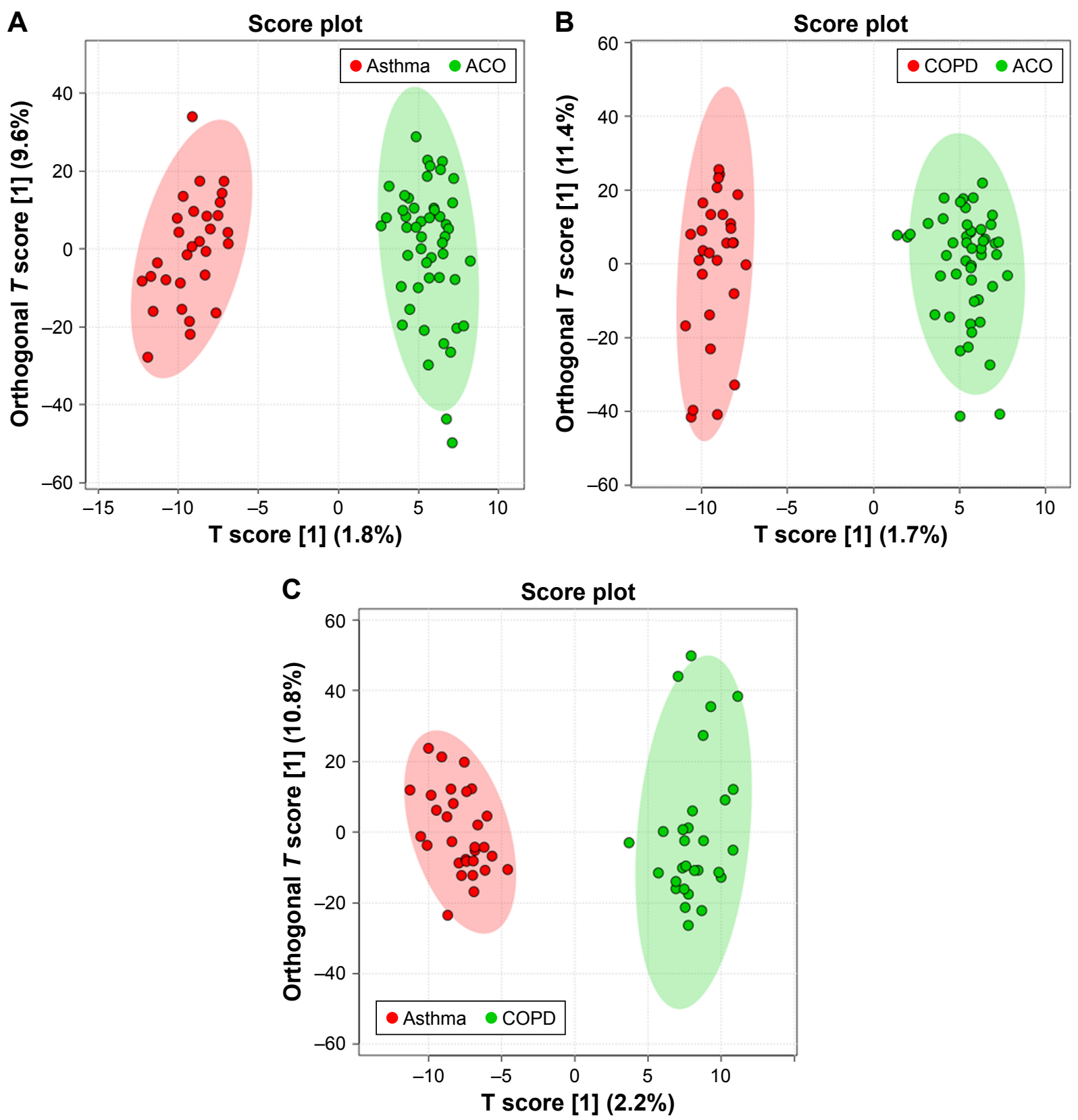

Figure I Differential urinary metabolic profiles in patients with asthma-COPD overlap (ACO), asthma, and COPD.

Notes: (A) Discrimination of the urine metabolome between patients with ACO and those with asthma by using orthogonal signal correction partial least squaresdiscriminant analysis (OPLS-DA). Intercepts: $R^{2}=0.964$ and $Q^{2}=0.153$. (B) Discrimination of the urine metabolome between patients with ACO and those with COPD by using OPLS-DA. Intercepts: $R^{2}=0.971$ and $Q^{2}=0.0466$. (C) Discrimination of the urine metabolome between patients with asthma and those with COPD by using OPLS-DA. Intercepts: $R^{2}=0.963$ and $Q^{2}=0.163$.

with asthma and COPD (Figure 2A). These results suggested that L-histidine was specifically elevated among patients with ACO.

\section{Urinary L-histidine in chronic airway disease}

Although the median urinary L-histidine levels were significantly higher in patients with ACO than in those with asthma or COPD (Figure 2A), no significant difference was observed in the levels of urinary L-histidine between smoking-related obstructive asthma $(\mathrm{n}=27)$ and COPD with a significantly positive BDR and/or blood eosinophilia (BDR $\geq 400 \mathrm{~mL}$ and/or blood eosinophil count $\geq 300$ cells $\cdot \mathrm{mm}^{-3}, \mathrm{n}=10$ ) (Figure 2B).

The overall sensitivity and specificity of ACO diagnosis were $75.7 \%$ and $63.0 \%$, respectively, at the urinary 
A

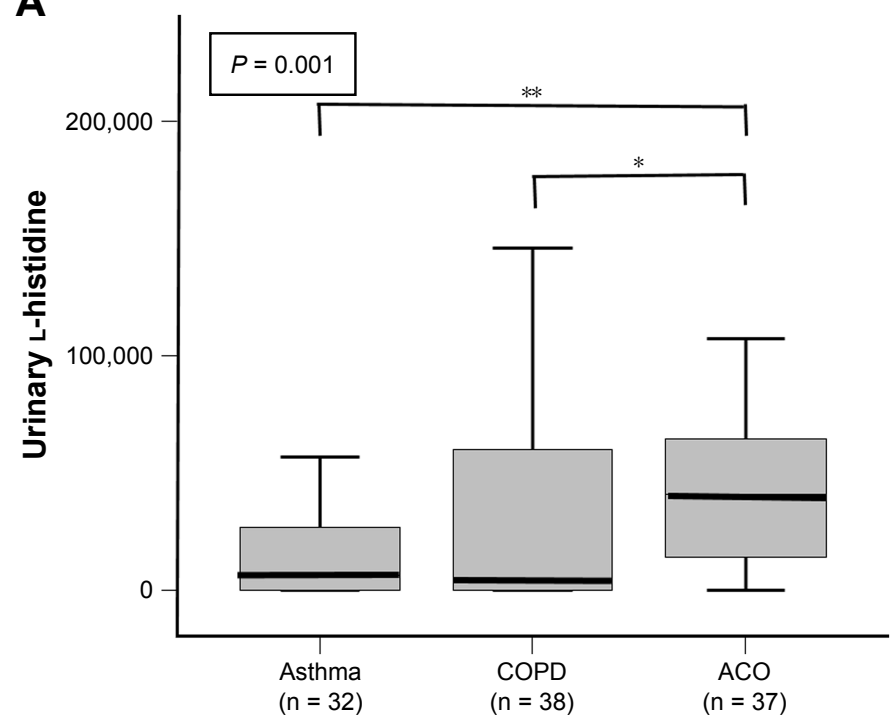

B

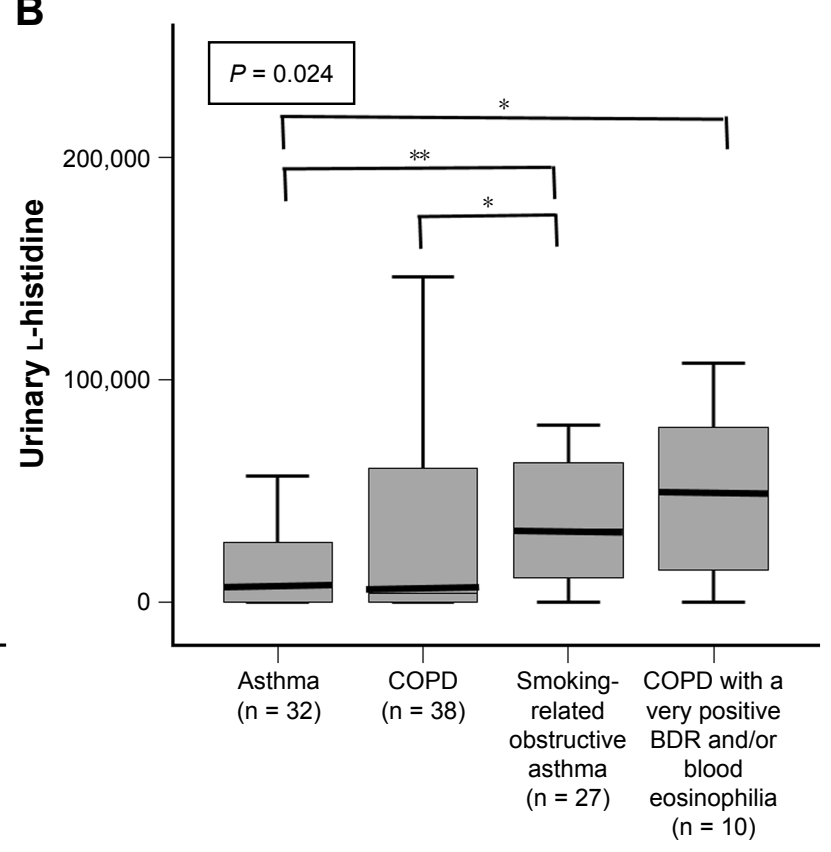

Figure 2 Urinary L-histidine levels in chronic inflammatory airway diseases according to disease classifications.

Notes: (A) Urinary L-histidine levels in patients with asthma, COPD, and ACO. (B) Urinary L-histidine levels in patients with asthma, COPD, smoking related obstructive asthma, and COPD with a very positive BDR and/or blood eosinophilia. $* P<0.05$, $* * P<0.01$.

Abbreviations: ACO, asthma-COPD overlap; BDR, bronchodilator response.

L-histidine cutoff of 13,860. Multivariate logistic regression revealed that a high urinary L-histidine level was a significant parameter of ACO even after adjusting for age, sex, and smoking amount (urinary L-histidine >13,860; OR, 5.254; 95\% CI, 2.085-13.236; $P<0.001)$.

\section{Urinary L-histidine levels and parameters}

In the total study population, urinary L-histidine levels were positively correlated with SGRQ scores $(\mathrm{R}=0.222 ; P=0.033)$ and negatively correlated with ACT scores $(\mathrm{R}=-0.284$; $P=0.024)$. In the PFTs, urinary L-histidine level was positively associated with $\mathrm{BDR}(\mathrm{R}=0.300 ; P=0.002)$, total lung capacity $(\mathrm{R}=0.242 ; P=0.012)$, and residual volume $(\mathrm{R}=0.263 ; P=0.006)$, and negatively correlated with $\mathrm{FEV}_{1}(\mathrm{R}=-0.220 ; P=0.022)$ and $\mathrm{FEV}_{1} / \mathrm{FVC}(\mathrm{R}=-0.228$, $P=0.018$ ) (Figure 3 ). There was no association between smoking amount and urinary L-histidine levels $(\mathrm{R}=0.001$, $P=0.990)$.

\section{Urinary L-histidine in COAD}

To evaluate the role of urinary L-histidine as a Th-2 inflammatory marker in patients with COAD without diagnostic labeling, we compared the urinary L-histidine levels between the following groups of patients: patients with and without a documented history of asthma before 40 years of age or a $\mathrm{BDR} \geq 400 \mathrm{~mL}$ of $\mathrm{FEV}_{1}$; patients with and without a $\mathrm{BDR} \geq 200 \mathrm{~mL}$ of $\mathrm{FEV}_{1}$ and exceeding the baseline values by $12 \%$ on two or more visits; and patients with high and low eosinophil counts (according to the experts' agreement regarding the Th-2 features of ACO). ${ }^{23}$ The levels of urinary L-histidine were significantly higher in patients with the previously mentioned characteristics than in those without these characteristics (Figure 4). Moreover, urinary L-histidine levels were significantly higher in frequent exacerbators than in non-frequent exacerbators (Table 2).

\section{Discussion}

This study investigated urinary L-histidine levels as a biomarker of ACO. Urinary L-histidine levels were elevated in patients with severe airflow obstruction, high BDR, severe symptoms, and exacerbation. Moreover, the levels were significantly higher in patients with key Th-2 features of ACO agreed upon by experts. ${ }^{23}$ Histidine is an essential amino acid that is a potential inflammatory metabolite, ${ }^{24}$ and it has been correlated with inflammation and oxidative stress in previous studies. ${ }^{25}$ Histidine is a precursor of histamine, which is important in the Th-2 inflammatory response and airway smooth muscle contraction. ${ }^{8,12,26}$ Thus, the current results imply that urinary L-histidine increases as smooth muscle contraction with reversibility increases and that urinary L-histidine could be an indicator of Th-2-related inflammation.

ACO has both features of asthma and COPD and is regarded to stem from either asthma or COPD. ${ }^{27}$ Miravitlles 

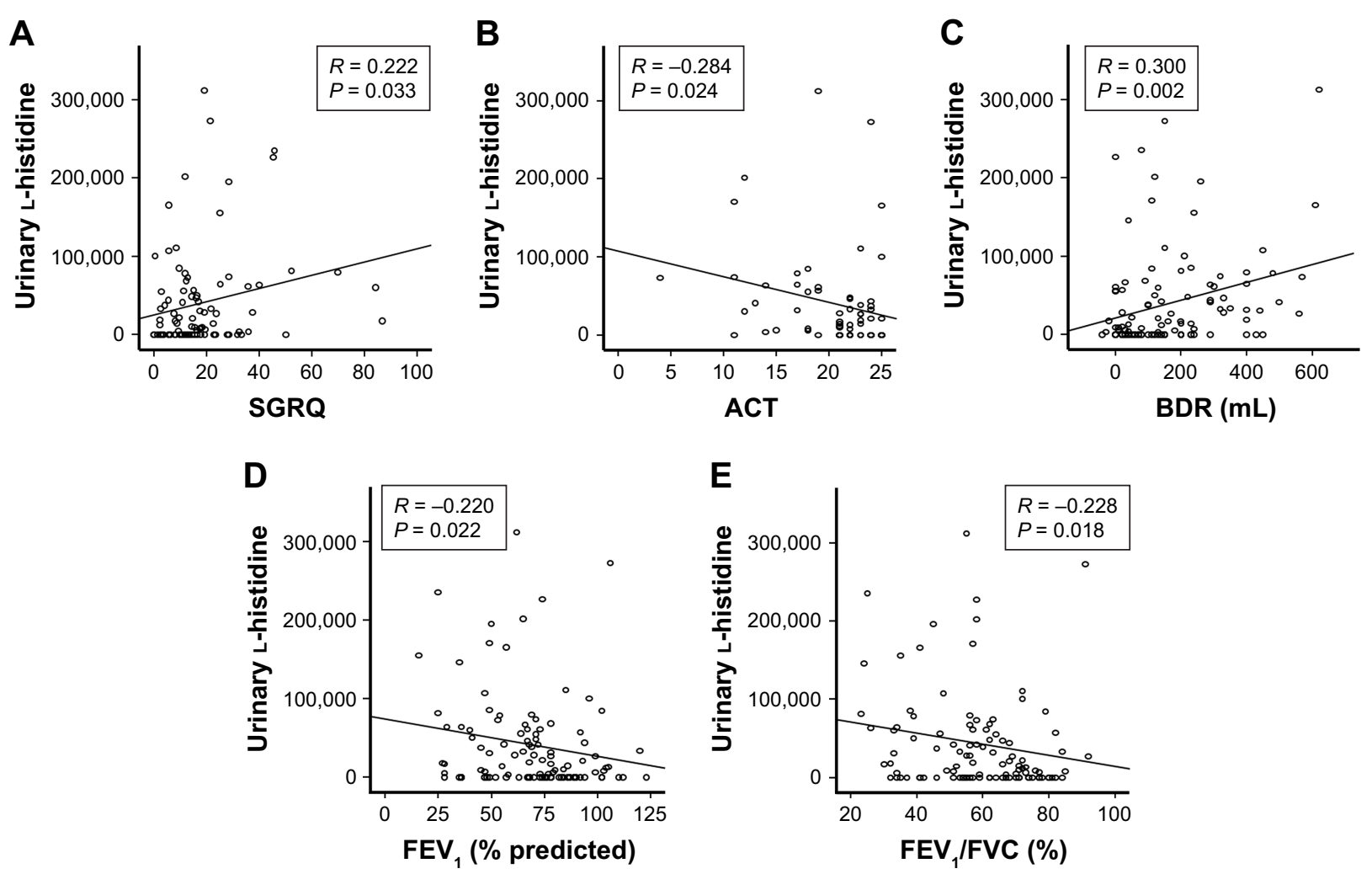

Figure 3 Correlation between urinary L-histidine levels and parameters.

Notes: (A) Correlation between urinary L-histidine levels and SGRQ. (B) Correlation between urinary L-histidine levels and ACT. (C) Correlation between urinary L-histidine levels and BDR (D) Correlation between urinary L-histidine levels and FEV, (E) Correlation between urinary L-histidine levels and FEV/FVC.

Abbreviations: ACT, asthma control test; BDR, bronchodilator response; FEV , forced expiratory volume in I second; FVC, forced vital capacity; RV, residual volume; SGRQ, St George Respiratory Questionnaire; TLC, total lung capacity.

has suggested that smoking-related obstructive asthma and COPD with a Th-2 genetic background or with significantly positive BDR could be classified as $\mathrm{ACO} .^{28}$ The former is regarded to originate from asthma, while the latter originates from COPD. However, when we divided patients with ACO into those with smoking-related obstructive asthma and COPD with a significantly positive BDR and/or blood eosinophilia groups, no significant differences were observed in urinary L-histidine levels between the two groups, even though other clinical characteristics, such as lung functions, were significantly different. The two subgroups of ACO are divided on the basis of different historic classifications, but this classification does not reflect inflammatory heterogeneity. ACO might be the different expressions of a single disease entity; this is because we frequently found difficulties in differentiating the origin between asthma and COPD. ${ }^{6} \mathrm{~A}$ recent study demonstrated that Th-2-related inflammatory gene expression was also up-regulated in COPD that could not be distinguished using a clinical history of asthma, ${ }^{7}$ and the Th-2-related signature provided a more clear distinction than the classical diagnosis. ${ }^{6}$ Moreover, in this study, if we applied the ACO criteria proposed by experts, ${ }^{23}$ we could classify only $48.1 \%$ of patients with smoking-related obstructive asthma and $40.0 \%$ of the patients with COPD with significantly positive BDR and/or high blood eosinophilia as having ACO. Therefore, the term "ACO" may be replaced when new phenotypes or endotypes are identified. ${ }^{2,27,29}$

Recently, emphasis has shifted from diagnostic labels to "treatable traits" among chronic airway inflammatory diseases. ${ }^{4}$ A primary goal in patients with COPD is to identify patients who may benefit from ICSs. ICS therapy can prevent exacerbation and improve the symptoms and health status of patients with COPD. ${ }^{30,31}$ However, the use of ICSs also carries the risks of osteoporosis, diabetes, and pneumonia. ${ }^{31,32}$ Therefore, it is important to find biomarkers that could guide the use of ICSs to reduce the risk-benefit ratio of ICS therapy.

In this study, urinary L-histidine levels were higher in patients identified using the recently proposed algorithm of $\mathrm{ACO}^{18}$ than in patients with asthma or COPD. However, the definition of and diagnostic criteria for ACO are variable, and thus, we removed the classical diagnosis in patients with airflow obstruction and compared the patients according to the characteristics - agreed upon by experts - related to Th-2 features in ACO (ie, a documented history of asthma before 40 years of age or a BDR $\geq 400 \mathrm{~mL}$ of $\mathrm{FEV}_{1}$, a 
A

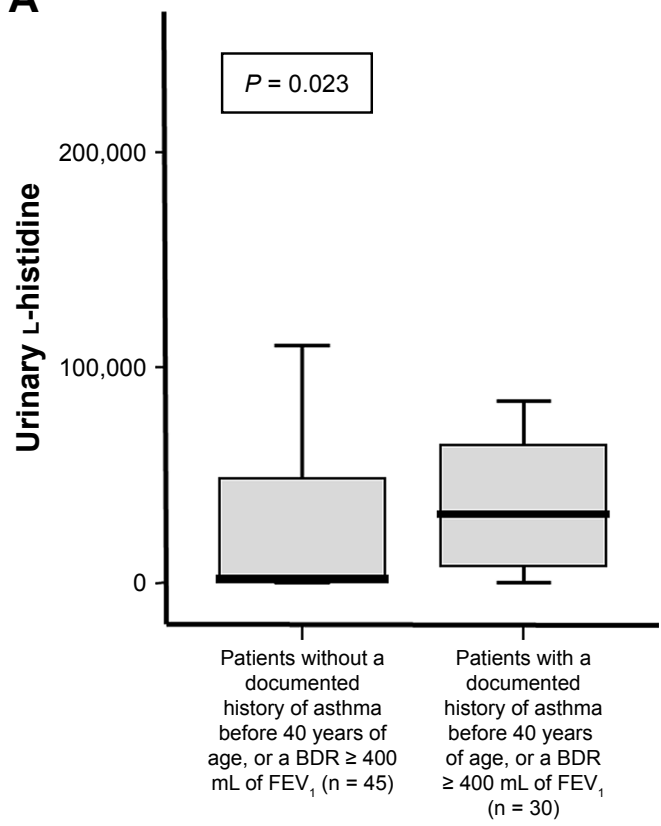

B

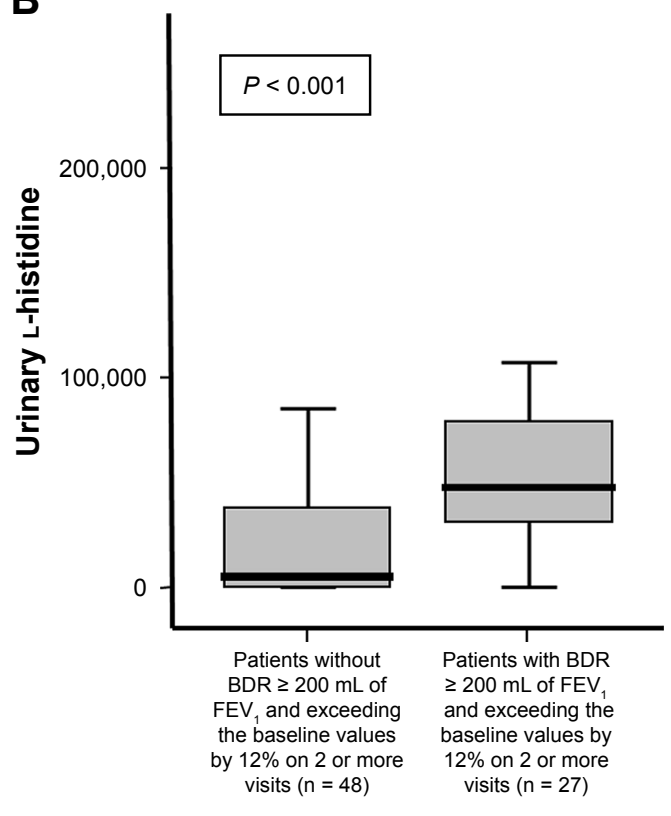

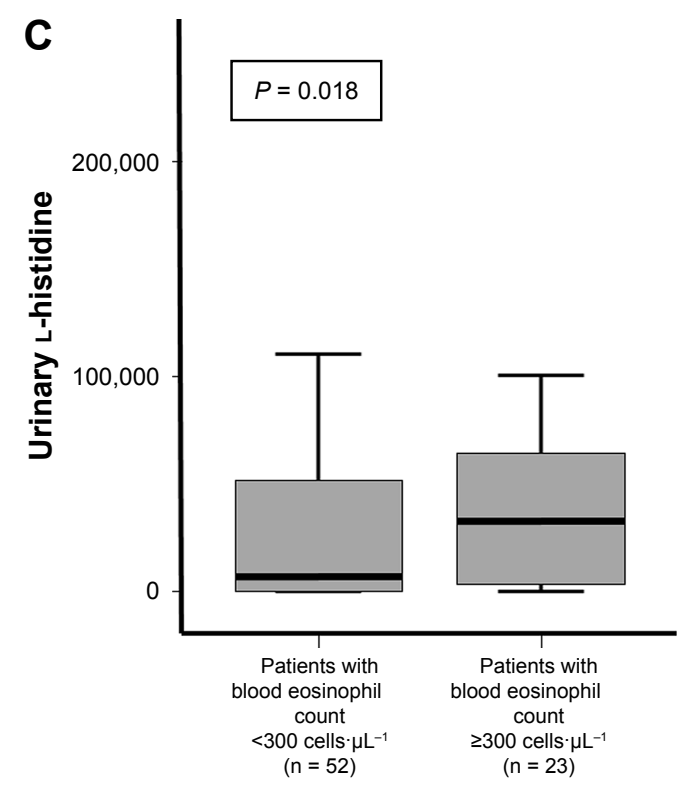

Figure 4 Urinary L-histidine levels for each category of patients with Th-2 features of ACO as agreed upon by experts.

Notes: (A) Urinary L-histidine levels in patients with and without a documented history of asthma before 40 years of age or a BDR $\geq \mathrm{mL}$ of FEV . (B) Urinary L-histidine levels in patients with and without $B D R \geq 200 \mathrm{~mL}$ of $F E V_{\text {, }}$ and exceeding the baseline values by $12 \%$ on 2 or more visits. (C) Urinary L-histidine levels in patients with blood eosinophil count $\geq 300$ cells $\mu \mathrm{L}^{-1}$ and $<300$ cells $\mu \mathrm{L}^{-1}$.

Abbreviations: ACO, asthma-COPD overlap; BDR, bronchodilator response; FEV , forced expiratory volume in I second.

$\mathrm{BDR} \geq 200 \mathrm{~mL}$ of FEV and exceeding baseline values by $12 \%$ on two or more visits, and a peripheral blood eosinophil count $\geq 300$ cells $\left.\cdot \mu \mathrm{L}^{-1}\right) .{ }^{23}$ Urinary L-histidine levels were significantly higher in patients with these characteristics. Thus, urinary L-histidine levels could be a biomarker for ACO, regardless of the diversity of ACO definitions.

In addition to considering Th-2 features, ICS use in patients with frequent exacerbation is still needed despite the recent trend of withdrawing ICS in patients with moderate-to- severe COPD without a history of exacerbation. ${ }^{5,33}$ A history of previous exacerbation is the most potent predictor of future exacerbation..$^{34-36}$ Thus, there is a need to perform ICS therapy in patients with previous frequent exacerbations despite treatment with bronchodilators. In this study, urinary L-histidine levels were also higher in frequent exacerbators, which supports the evidence indicating that urinary L-histidine is a candidate biomarker for guiding the decision to initiate ICS therapy. 
Table 2 Comparison between frequent exacerbator and nonfrequent exacerbator

\begin{tabular}{|c|c|c|c|}
\hline Parameters & $\begin{array}{l}\text { Frequent } \\
\text { exacerbator } \\
n=12\end{array}$ & $\begin{array}{l}\text { Non-frequent } \\
\text { exacerbator } \\
n=95\end{array}$ & $P$-value \\
\hline Age, years & $61.5(54.3-72.3)$ & $64.0(57.0-70.0)$ & 0.667 \\
\hline Sex, male & $10(83.3 \%)$ & $72(75.8 \%)$ & 0.547 \\
\hline Smoker & $10(83.3 \%)$ & $64(67.3 \%)$ & 0.010 \\
\hline Ex-smoker & 9 (75.0\%) & 35 (36.8\%) & \\
\hline Current smoker & I (8.3\%) & $29(30.5 \%)$ & \\
\hline $\begin{array}{l}\text { Smoking amount } \\
\text { (pack-years) }\end{array}$ & $37.5(12.5-48.8)$ & $40.0(33.8-54.8)$ & 0.301 \\
\hline $\begin{array}{l}\text { Urine L-histidine } \\
\text { level }\end{array}$ & $\begin{array}{l}61,917.8 \\
(6,200.0-153,053.1)\end{array}$ & $\begin{array}{l}\text { I2,226.3 } \\
(0.0-48,566.3)\end{array}$ & 0.032 \\
\hline
\end{tabular}

Note: Data are presented as median interquartile range for continuous variables and percentage (number) for categorical variables.

Despite its strengths, this study had some limitations. First, because this study was based on urine analysis, the results could be affected by the time of urine collection. However, we collected urine samples after overnight fasting and in a medication-free state when patients were stable without exacerbation for 3 months. Moreover, we checked the metabolites three times and used the mean results. No patients had renal diseases, and the creatinine clearance rates of all enrolled patients were within the normal range. Furthermore, different from other parameters such as blood markers, urine has an advantage that its sampling is non-invasive and easy. Second, a quantitative analysis was not performed for urine metabolites. Further studies including quantitative analysis and proteomics/genomics studies are necessary to confirm the diagnostic value of urinary L-histidine and to elucidate the underlying mechanism.

\section{Conclusion}

Urinary L-histidine could be a potential biomarker for ACO and could be an adjunctive biomarker for ICS guidance, regardless of the diagnosis.

\section{Acknowledgment}

We thank SY Hwang for the excellent statistical support. AstraZeneca supported this study.

\section{Author contributions}

All authors contributed toward data analysis, drafting and revising the paper and agree to be accountable for all aspects of the work.

\section{Disclosure}

The authors report no conflicts of interest in this work.

\section{References}

1. Wedzicha JA, Price D, Mezzi K, Fogel R, Banerji D. QVA149 compared with salmeterol/fluticasone (SFC) on exacerbations and its correlation with baseline blood eosinophils: a pooled analysis of LANTERN and ILLUMINATE. Eur Respir J. 2015;46:PA1005.

2. Cazzola M, Rogliani P. Do we really need asthma-chronic obstructive pulmonary disease overlap syndrome? J Allergy Clin Immunol. 2016; 138(4):977-983.

3. Turner AM, Tamasi L, Schleich F, et al. Clinically relevant subgroups in COPD and asthma. Eur Respir Rev. 2015;24(136):283-298.

4. Agusti A, Bel E, Thomas M, et al. Treatable traits: toward precision medicine of chronic airway diseases. Eur Respir J. 2016;47(2): 410-419.

5. Kaplan AG. Applying the wisdom of stepping down inhaled corticosteroids in patients with COPD: a proposed algorithm for clinical practice. Int J Chron Obstruct Pulmon Dis. 2015;10:2535-2548.

6. Cosío BG, Perez de Llano L, Lopez Viña A, et al. Th-2 signature in chronic airway diseases: towards the extinction of asthma-COPD overlap syndrome? Eur Respir J. 2017;49(5):1602397.

7. Christenson SA, Steiling K, van den Berge M, et al. Asthma-COPD overlap. Clinical relevance of genomic signatures of type 2 inflammation in chronic obstructive pulmonary disease. Am J Respir Crit Care Med. 2015;191(7):758-766.

8. Jung J, Kim SH, Lee HS, et al. Serum metabolomics reveals pathways and biomarkers associated with asthma pathogenesis. Clin Exp Allergy. 2013;43(4):425-433.

9. Hirst SJ, Lee TH. Airway smooth muscle as a target of glucocorticoid action in the treatment of asthma. Am J Respir Crit Care Med. 1998; 158(5 Pt 3):S201-S206.

10. Hospers JJ, Postma DS, Rijcken B, Weiss ST, Schouten JP. Histamine airway hyper-responsiveness and mortality from chronic obstructive pulmonary disease: a cohort study. Lancet. 2000;356(9238):1313-1317.

11. White MV, Slater JE, Kaliner MA. Histamine and asthma. Am Rev Respir Dis. 1987;135(5):1165-1176.

12. Mazzoni A, Young HA, Spitzer JH, Visintin A, Segal DM. Histamine regulates cytokine production in maturing dendritic cells, resulting in altered T cell polarization. J Clin Invest. 2001;108(12):1865-1873.

13. Forsythe IJ, Wishart DS. Exploring human metabolites using the human metabolome database. Curr Protoc Bioinformatics. 2009; Chapter 14: Unit14.8.

14. Bouatra S, Aziat F, Mandal R, et al. The human urine metabolome. PLoS One. 2013;8(9):e73076.

15. Wishart DS. Emerging applications of metabolomics in drug discovery and precision medicine. Nat Rev Drug Discov. 2016;15(7):473-485.

16. Global Initiative for Chronic Obstructive Lung Disease (GOLD) [webpage on the Internet]. Global Strategy for Diagnosis, Management and Prevention of COPD 2017. GOLD; 2017. Available from: http://goldcopd.org/gold-2017-global-strategy-diagnosis-managementprevention-copd/. Accessed April 9, 2018.

17. Global Initiative for Asthma [webpage on the Internet]. 2017 GINA Report, Global Strategy for Asthma Management and Prevention. GINA; 2017. Available from: http://ginasthma.org/gina-reports/. Accessed April 9, 2018.

18. Miravitlles M, Alvarez-Gutierrez FJ, Calle M, et al. Algorithm for identification of asthma-COPD overlap: consensus between the Spanish COPD and asthma guidelines. Eur Respir J. 2017;49(5). pii: 1700068.

19. Johnson JM, Yu T, Strobel FH, Jones DP. A practical approach to detect unique metabolic patterns for personalized medicine. Analyst. 2010;135(11):2864-2870.

20. Pamungkas AD, Park C, Lee S, Jee SH, Park YH. High resolution metabolomics to discriminate compounds in serum of male lung cancer patients in South Korea. Respir Res. 2016;17(1):100.

21. Yu T, Park Y, Johnson JM, Jones DP. apLCMS - adaptive processing of high-resolution LC/MS data. Bioinformatics. 2009;25(15):1930-1936.

22. Wishart DS, Jewison T, Guo AC, et al. HMDB 3.0 - the human metabolome database in 2013. Nucleic Acids Res. 2012;41(Database issue): D801-D807. 
23. Sin DD, Miravitlles M, Mannino DM, et al. What is asthma-COPD overlap syndrome? Towards a consensus definition from a round table discussion. Eur Respir J. 2016;48(3):664-673.

24. Andersen HH, Elberling J, Arendt-Nielsen L. Human surrogate models of histaminergic and non-histaminergic itch. Acta Derm Venereol. 2015;95(7):771-779.

25. Gervasini G, Agúndez JA, García-Menaya J, et al. Variability of the L-Histidine decarboxylase gene in allergic rhinitis. Allergy. 2010;65(12): 1576-1584.

26. Caron G, Delneste Y, Roelandts E, et al. Histamine polarizes human dendritic cells into Th2 cell-promoting effector dendritic cells. J Immunol. 2001;167(7):3682-3686.

27. Postma DS, Rabe KF. The asthma-COPD overlap syndrome. $N$ Engl J Med. 2015;373(13):1241-1249.

28. Miravitlles M. Diagnosis of asthma-COPD overlap: the five commandments. Eur Respir J. 2017;49(5):1700506.

29. Barrecheguren M, Esquinas C, Miravitlles M. The asthma-COPD overlap syndrome: a new entity? COPD Research and Practice. 2015; 1(1):8.

30. Suissa S, McGhan R, Niewoehner D, Make B. Inhaled corticosteroids in chronic obstructive pulmonary disease. Proc Am Thorac Soc. 2007;4(7):535-542.
31. Price D, Yawn B, Brusselle G, Rossi A. Risk-to-benefit ratio of inhaled corticosteroids in patients with COPD. Prim Care Respir J. 2013;22(1): 92-100.

32. Suissa S, Patenaude V, Lapi F, Ernst P. Inhaled corticosteroids in COPD and the risk of serious pneumonia. Thorax. 2013;68(11):1029-1036.

33. Rossi A, Guerriero M, Corrado A; OPTIMO/AIPO Study Group. Withdrawal of inhaled corticosteroids can be safe in COPD patients at low risk of exacerbation: a real-life study on the appropriateness of treatment in moderate COPD patients (OPTIMO). Respir Res. 2014; 15:77.

34. Santibáñez M, Garrastazu R, Ruiz-Nuñez M, et al. Predictors of hospitalized exacerbations and mortality in chronic obstructive pulmonary disease. PLoS One. 2016;11(6):e0158727.

35. McGhan R, Radcliff T, Fish R, et al. Predictors of rehospitalization and death after a severe exacerbation of COPD. Chest. 2007;132(6): $1748-1755$

36. Hurst JR, Vestbo J, Anzueto A, et al. Susceptibility to exacerbation in chronic obstructive pulmonary disease. New Engl J Med. 2010;363(12): 1128-1138.
International Journal of COPD

\section{Publish your work in this journal}

The International Journal of COPD is an international, peer-reviewed journal of therapeutics and pharmacology focusing on concise rapid reporting of clinical studies and reviews in COPD. Special focus is given to the pathophysiological processes underlying the disease, intervention programs, patient focused education, and self management protocols.

\section{Dovepress}

This journal is indexed on PubMed Central, MedLine and CAS. The manuscript management system is completely online and includes a very quick and fair peer-review system, which is all easy to use. Visit http://www.dovepress.com/testimonials.php to read real quotes from published authors. 